\title{
Variability in estimating shunt from single pulse oximetry measurements
}

\section{Journal Article}

\section{Author(s):}

Karlen, Walter (D); Petersen, Christian L.; Dumont, Guy A.; Ansermino, J. Mark

Publication date:

2015-05

Permanent link:

https://doi.org/10.3929/ethz-b-000100538

Rights / license:

In Copyright - Non-Commercial Use Permitted

Originally published in:

Physiological Measurement 36(5), https://doi.org/10.1088/0967-3334/36/5/967 


\title{
Variability in Estimating Shunt from Single Pulse Oximetry Measurements
}

\author{
Walter Karlen*, Christian L Petersen ${ }^{\dagger}$, Guy A Dumont ${ }^{\ddagger}$, J Mark Ansermino ${ }^{\dagger}$ \\ * Department of Health Sciences and Technology, \\ ETH Zurich, \\ Zurich, Switzerland \\ $\dagger$ Department of Anesthesiology, Pharmacology and Therapeutics, \\ The University of British Columbia, \\ Vancouver, BC, Canada \\ $\ddagger$ Department of Electrical and Computer Engineering, \\ The University of British Columbia, \\ Vancouver, BC, Canada \\ walter.karlen@ieee.org
}

Corrected version: 26.6.2017 


\begin{abstract}
Virtual shunt describes the overall loss of $\mathrm{O}_{2}$ content between the alveolar gas and arterial blood. Clinicians indirectly estimate the magnitude of the virtual shunt by monitoring peripheral blood oxygen saturation $\left(\mathrm{SpO}_{2}\right)$ using non-invasive pulse oximetry. An inherent limitation of this method is the variable precision of pulse oximeters and the non-linear relationship between virtual shunt and $\mathrm{SpO}_{2}$ which is rarely depicted.

We propose a model using a combination of basic physiological equations to analyze the estimation of virtual shunt from inspired oxygen $\left(\mathrm{FiO}_{2}\right)$ and $\mathrm{SpO}_{2}$. The model emphasizes the effect of the non-linearity of the $\mathrm{Hb}-\mathrm{O}_{2}$ dissociation curve. Furthermore, it accounts for the variability in $\mathrm{SpO}_{2}$ measurements due to the precision of pulse oximeters.

The model was validated with experiments conducted on healthy subjects in a normobaric hypoxia chamber comparing the simultaneous readings from two different commercial pulse oximeters at $\mathrm{FiO}_{2}$ $=21 \%$ and $17 \% . \mathrm{SpO}_{2}$ probability distributions calculated from the model were estimated. Although a variable bias (1.2\% to $2.1 \%$ ) in $\mathrm{SpO}_{2}$ between the pulse oximeter brands was observed, the tested pulse oximeters were both within tolerance specified by the manufacturers and matched the probability distributions from the model.

The theoretical and experimental findings show that the estimation of virtual shunt is challenging with a single $\mathrm{SpO}_{2}$ measurement using pulse oximeters with tolerances of $2 \%$. Clinical decisions must be based on an appreciation of these limitations.

Keywords: Pulse oximeter, Accuracy, Tolerance, Modeling, Oxygen Cascade, Shunt, Pulmonary Gas Exchange
\end{abstract}




\section{Introduction}

Pulse oximeters have become essential noninvasive monitoring devices in operating rooms and intensive care units for measuring the peripheral oxygen saturation $\left(\mathrm{SpO}_{2}\right)$ and heart rate. The clinician is reassured by an $\mathrm{SpO}_{2}$ of $98 \%$ to $100 \%$ when the patient is breathing room air at sea level. Concerns arise when the $\mathrm{SpO}_{2}$ is less than $96 \%$ or drops from $99 \%$ to $96 \%$ or more in a healthy patient. The technical limitations and environmental effects (such as altitude) of the measurement of $\mathrm{SpO}_{2}$ must be appreciated by the clinician to ensure appropriate clinical decision making. For example, $\mathrm{SpO}_{2}$ of $99 \%$ or $100 \%$ are not physiologically plausible in a healthy subject breathing room air oxygen concentrations. This is due to the oxygen affinity of hemoglobin and the resulting hemoglobin-oxygen $\left(\mathrm{Hb}-\mathrm{O}_{2}\right)$ dissociation curve (West 2012). This frequent overestimation of high $\mathrm{SpO}_{2}$ is attributed to measurement tolerances of pulse oximeters and is device and brand specific (Webb et al. 1991).

An inherent limitation of pulse oximeters is the relatively large tolerance intervals $( \pm 2-3 \%)$ leading to a low precision of the arterial blood oxygen saturation $\left(\mathrm{SaO}_{2}\right)$ estimation. Technical limitations of pulse oximeters and subject variability contribute to these large measurement inaccuracies. The agreement of measurements is affected by light-tissue interactions, sensor manufacturing variations, manufacturer specific in vivo calibrations, and variations in application and handling of the probe. All of these factors can introduce errors in the $\mathrm{SaO}_{2}$ estimation, potentially leading to inadequate intervention (Jubran 1999). Knowledge of the accuracy of the performed estimation is essential for appropriate clinical decision making (Weininger 2007). To assess the reliability of the provided measure, clinicians must be aware of the device limitations.

Device specification standards demand reporting the expected accuracy of the $\mathrm{SpO}_{2}$ reading in the device manual (ISO 2011). However, in clinical diagnostic situations, a binary decision is frequently required (normal or abnormal $\mathrm{SpO}_{2}$ ). For example, when determining the presence of hypoxemia in a critically ill patient, a fixed classification threshold of $90 \%$ is typically used, but with the observed inaccuracy in the estimation, thresholds of up to $94 \%$ have been suggested (Van de Louw et al. 2001). In such situations an inaccurate knowledge of the $\mathrm{SaO}_{2}$ can severely under- or overestimate the severity of the underlying disease process and lead to inaccurate treatments (Van de Louw et al. 2001).

Virtual shunt is a theoretical concept that describes the overall loss of $\mathrm{O}_{2}$ content between the alveolar gas and arterial blood (West 2012). Estimating virtual shunt is of importance to the clinician as the magnitude of shunt describes the efficiency of the gas exchange and severity of a disease process causing a reduction in oxygenation. Shunt is measured in the clinical setting using a pulmonary artery catheter. However, in practice this is rarely available. Instead, virtual shunt is typically estimated from oxygen partial pressure measurements of the arterial blood $\left(\mathrm{PaO}_{2}\right)$, obtained from blood gas analysis. Since $\mathrm{SpO}_{2}$ obtained from pulse oximetry is an estimate of the $\mathrm{SaO}_{2}$, the magnitude of virtual shunt could be indirectly estimated using this non-invasive device. However, in clinical applications where individual measurements are performed and a single $\mathrm{SpO}_{2}$ measure is taken, the wide distribution of possible $\mathrm{SaO}_{2}$ values should be appreciated when estimating virtual shunt.

Portable pulse oximeters are increasingly used as spot-check devices where sampling of arterial blood is not available. The availability of very few data points poses an increased challenge in the estimation 
of virtual shunt. In this study we describe a model to estimate virtual shunt from pulse oximeter readings. The model illustrates the impact of accuracy of pulse oximeters. We provide a mathematical, physiological framework to describe the propagation of specified tolerances of pulse oximeter readings in the estimation of oxygen saturation across the different stages of the oxygen cascade used for the estimation of the virtual shunt. The estimation of the $\mathrm{SaO}_{2}$ value based on measured $\mathrm{SpO}_{2}$ readings and the oxygen cascade has application in automated decision making algorithms. Providing the level of certainty (tolerance intervals) with each measurement may help increase awareness among medical personnel of limitations in pulse oximeter accuracy.

\subsection{Background}

The fundamental principle of pulse oximetry is a light-tissue interaction. The absorption of light by tissue depends on the wavelength, the tissue composition and the distance (optical path) traveled by the light which changes with each heart beat as blood volume in vessels changes. $\mathrm{SaO}_{2}$ describes the percentage of oxygenated hemoglobin over the total functional hemoglobin and can be estimated through measuring the ratio $(\mathrm{R})$ of pulsatile and nonpulsatile components of the nonabsorbed light at two distinct wavelengths (typically $660 \mathrm{~nm}$ and $910 \mathrm{~nm}$ ). The relationship between $\mathrm{R}$ and $\mathrm{SaO}_{2}$ can be theoretically explained with the Beer-Lambert law. However this description ignores scattering of light (Mannheimer 2007) and various other design aspects of oximeters, such as optical shunt or probe type. To account for these effects, the relationship between $\mathrm{R}$ and $\mathrm{SpO}_{2}$ is empirically determined by pulse oximeter manufacturers for each device. Even with empirical calibration, $\mathrm{SpO}_{2}$ remains only an estimate of $\mathrm{SaO}_{2}$.

The design of pulse oximeters is currently regulated by the International Standards Organization (ISO) specification ISO 80601-2-61 (ISO 2011). This standard describes the requirements for the design of pulse oximeters such as packaging, electro-magnetic safety, alarms, and display. Further, the standards give recommendations on the performance of calibration experiments using desaturation studies with human subjects, including testing and reporting accuracy. Manufacturers are required to specify the accuracy for each device and sensor combination since each configuration requires a different transfer function relating $\mathrm{R}$ to $\mathrm{SaO}_{2}$. This accuracy is generally stated as root mean square error $\left(\mathrm{A}_{R M S}\right)$ between measured and reference values obtained during desaturation studies such as

$$
A_{R M S}=\sqrt{\frac{\sum_{i=1}^{n}\left(S p O_{2}^{i}-S a O_{2}^{i}\right)^{2}}{n}}
$$

where $\mathrm{n}$ is the number of observations and $\mathrm{SaO}_{2}$ is the arterial saturation obtained from a reference carboxy-hemoglobin oximeter (CO-oximeter or hemoximeter) with an accuracy of $\leq 1 \%$. It is important to note that hemoximeter devices are also subject to a measurement bias between models (Gehring et al. 2007). In pulse oximeter data sheets and manufacturer documentation, which are generally available to clinicians, the $\mathrm{A}_{R M S}$ value is often incorrectly referred to as 'standard deviation' (SD) (ISO 2011). According to the ISO standard, the bias $B_{S}$ and the precision $P_{S}$ of $\mathrm{SpO}_{2}$ can also be 

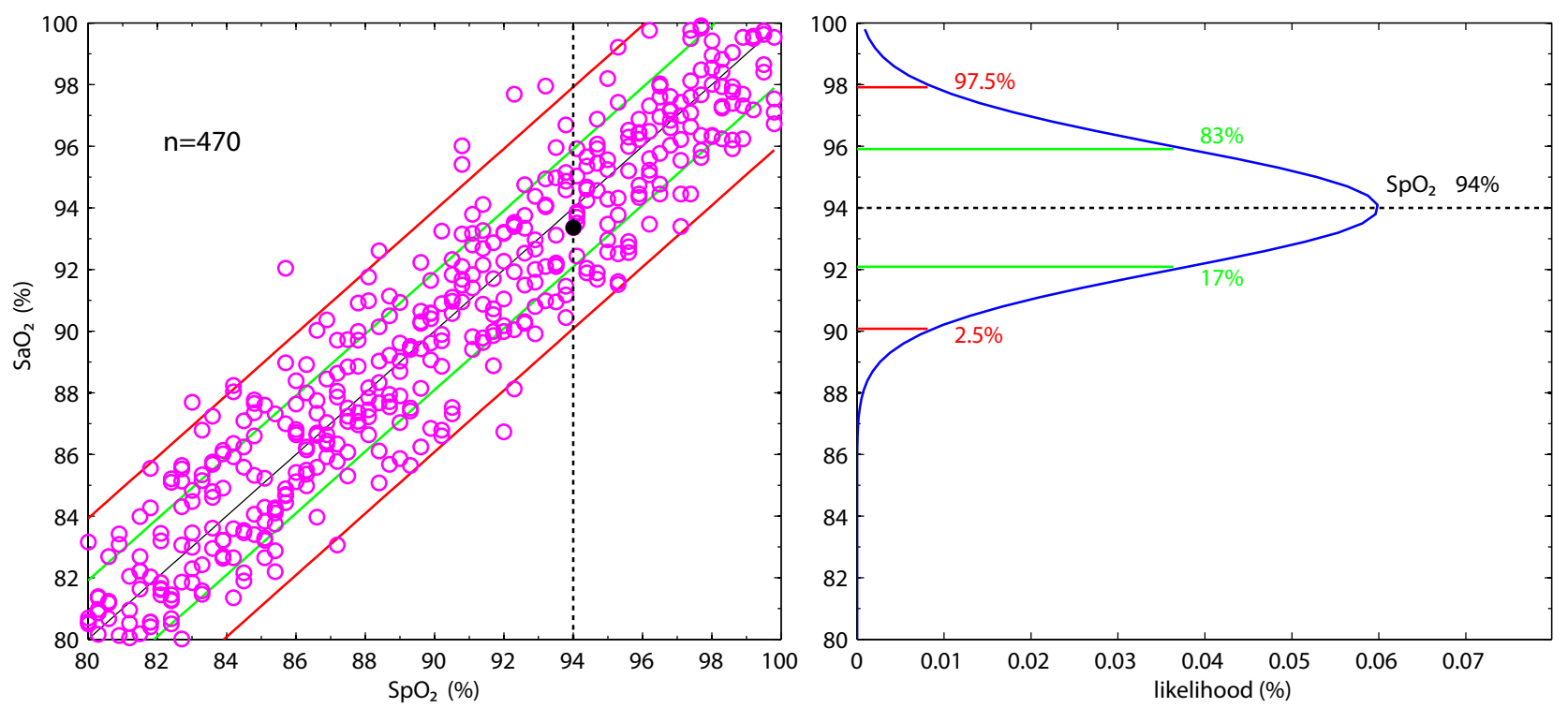

Figure 1: Simulated relationship between oxygen saturation measured by a pulse oximeter $\left(\mathrm{SpO}_{2}\right)$ and arterial blood $\left(\mathrm{SaO}_{2}\right)$. Left: Scatter of simulated $\mathrm{SaO}_{2}$ for $470 \mathrm{SpO}_{2}$ values from a theoretical pulse oximeter with an accuracy of $\mathrm{A}_{R M S}=2 \%$. Right: Probability density function of $\mathrm{SaO}_{2}$ for a pulse oximeter measurement of $\mathrm{SpO}_{2}=94 \%\left(\mathrm{~A}_{R M S}=2 \%\right)$. The pulse oximeter tolerances (confidence intervals of accuracy) are represented by the green (68\%) and red (95\%) lines.

used to describe device errors such as

$$
A_{R M S} \approx \sqrt{P s^{2}+B s^{2}}
$$

If $B_{S}$ is close to $0, \mathrm{~A}_{R M S}=P_{S}$ (ISO 2011). In this case the mean $S p O_{2} \approx \mathrm{SaO}_{2}$ and $\mathrm{A}_{R M S} \approx S D$. Consequently, $\mathrm{A}_{R M S}$ indirectly describes the confidence interval of finding a $\mathrm{SaO}_{2}$ for a given $\mathrm{SpO}_{2}$ $\pm \mathrm{A}_{R M S}$, where approximately $32 \%$ of measurements will be expected to be outside of this range. It can therefore be seen as a tolerance that describes an acceptable deviation from the target value. The $\mathrm{A}_{R M S}$ error can be represented as a Gaussian distribution with a center value corresponding to the observed $\mathrm{SpO}_{2}$ (Figure 1).

A deviation of $<4 \%$ has been considered acceptable for pulse oximeters used for monitoring (ISO 2011). Commercial pulse oximeters used in current clinical practice (e.g. monitors from brands like Nellcor, Nonin, Masimo and their OEM partners) have $\mathrm{A}_{R M S}$ between 1\% and 3\%, depending on the testing condition $\left(\mathrm{SaO}_{2}\right.$ range, probe type and presence of artifacts). Under non-artifact conditions and normal $\mathrm{SaO}_{2}$, an $\mathrm{A}_{R M S}$ of $2 \%$ is common. The $\mathrm{SaO}_{2}$ estimation of a pulse oximeter with this specification will have a $95 \%$ tolerance interval between $90 \%$ and $98 \%$ when displaying an $\mathrm{SpO}_{2}$ reading of $94 \%$, if we hypothesize that the bias of the estimation is low $(B s \approx 0)$ (Figure 1). This relatively large 
range of $8 \%$ around this common decision threshold of $94 \%$ might be clinically acceptable for continuous monitoring applications where many measurement points are available and trend changes of vital signs are of more interest than absolute values. However, in diagnosis and treatment applications where individual measurements are performed (so called spot-checks), this wide distribution of possible $\mathrm{SaO}_{2}$ should be taken into account when making clinical decisions. The clinical decision could be supported with a clinically relevant physiological mathematical model that accounts for the inherent inaccuracies of the measuring device.

Mathematical descriptions of the physiology of the human respiratory system and the propagation of $\mathrm{O}_{2}$ from inspiration at the lung to consumption at the cell level are well described (West 2012). Formulae to calculate iso-shunt lines for estimation of optimal oxygen administration (Benatar et al. 1973, Petros et al. 1994), or for predicting arterial oxygen partial pressures $\left(\mathrm{PaO}_{2}\right)$ from administered oxygen concentrations (Kretschmer et al. 2013) have been investigated and implemented in clinical practice. Pulse oximetry data have however frequently been used interchangeably with $\mathrm{SaO}_{2}$ in these implementations (Jones \& Jones 2000). In Kjaergaard et al. (2003), no significant difference in shunt estimation was observed when using either $\mathrm{SaO}_{2}$ or $\mathrm{SpO}_{2}$. However, in addition to $\mathrm{SaO}_{2}$ the model required measurement of expired $\mathrm{O}_{2}$ and did not take into account the precision of pulse oximeters.

In the next sections we describe the development of a mathematical model to describe the propagation of specified tolerances of pulse oximeter readings for the estimation of the virtual shunt (VS), an indicator of the severity of the gas exchange impairment in maximizing oxygen content. We then illustrate the impact of the theoretical concepts with controlled experimental data. In the discussion, we provide a critical analysis of advantages and limitations of the suggested model and the performed experiments.

\section{Methods}

We developed a model of the oxygen cascade through different stages of the body, $\mathrm{x}$, with a theoretically calculated oxygen partial pressures $\left(\mathrm{PxO}_{2}\right)$ at the inspired gas $\left(\mathrm{PiO}_{2}\right)$, alveolar $\left(\mathrm{PAO}_{2}\right)$, capillary $\left(\mathrm{PcO}_{2}\right)$, and systemic arterial stages (Figure 2). The $\mathrm{PxO}_{2}$ gradients at these stages were then graphically represented in an oxygen cascade graph. The difference between $\mathrm{PAO}_{2}$ and $\mathrm{PaO}_{2}\left(\mathrm{P}[\mathrm{A}-\mathrm{a}] \mathrm{O}_{2}\right)$ indicates the severity of the gas exchange abnormality. To estimate $\mathrm{P}[\mathrm{A}-\mathrm{a}] \mathrm{O}_{2}$, we calculated $\mathrm{PAO}_{2}$ at a known inspired oxygen concentration for the ideal lung and $\mathrm{PaO}_{2}$ from the $\mathrm{Hb}-\mathrm{O}_{2}$ dissociation relationship with $\mathrm{SaO}_{2}$ and assumed this to be equivalent to the $\mathrm{SpO}_{2}$ obtained from a pulse oximeter. We then analyzed the effects of pulse oximeter $\mathrm{A}_{R M S}$ on $\mathrm{P}[\mathrm{A}-\mathrm{a}] \mathrm{O}_{2}$ and VS. These theoretical results were then compared with pulse oximetry data obtained from healthy subjects. The experiment data were used to demonstrate the variability of the pulse oximeter measurements, to confirm the Gaussian distribution of pulse oximeter values at normal and hypoxic levels, and highlight the implications for clinical decision making. 


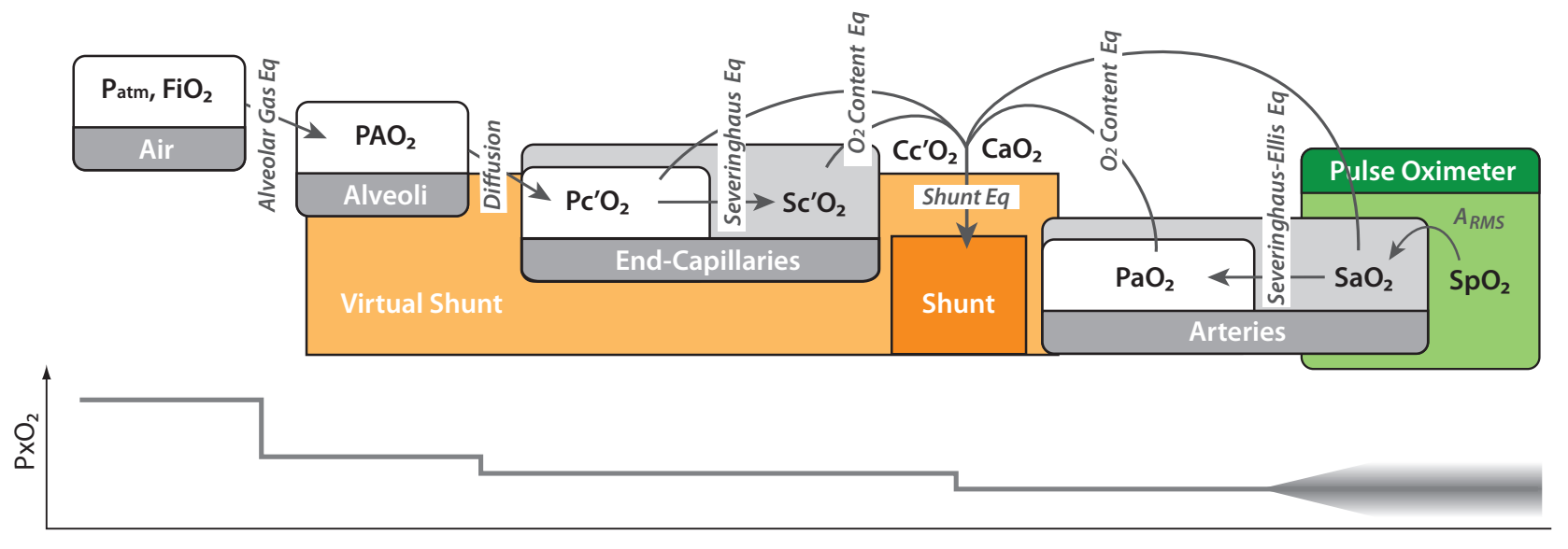

Figure 2: Theoretical representation of the oxygen cascade from inspired gas (Air) to pulse oximeter. Top: $\mathrm{O}_{2}$ partial pressures $\left(\mathrm{PxO}_{2}\right)$ are calculated for alveolar $(\mathrm{x}=\mathrm{A})$ gas, end-capillary $(\mathrm{x}=\mathrm{c})$ blood and arterial $(\mathrm{x}=\mathrm{a})$ blood with basic physiological equations (arrows) from the fraction of inspired $\mathrm{O}_{2}$ $\left(\mathrm{FiO}_{2}\right)$ and a pulse oximeter blood saturation measurement $\left(\mathrm{SpO}_{2}\right)$. The shunt equation estimates virtual shunt (VS) using $\mathrm{PxO}_{2}$ and the corresponding oxygen saturations $\mathrm{SxO}_{2}$ to calculate oxygen content $\left(\mathrm{CxO}_{2}\right)$. Arterial oxygen saturation $\left(\mathrm{SaO}_{2}\right)$ is estimated with an accuracy of $\mathrm{A}_{R M S}$ from $\mathrm{SpO}_{2}$. Bottom: Illustration of $\mathrm{O}_{2}$ transfer and $\mathrm{PxO}_{2}$ depletion at interfaces between different compartments. $\mathrm{A}_{R M S}$ of pulse oximeters is represented with a spreading of the $\mathrm{PaO}_{2}$ line and gradient coloring. 


\subsection{Theoretical Calculations}

The Alveolar Gas Equation (West 2012) with a fixed respiratory quotient (RQ), water vapor pressure $\left(\mathrm{PH}_{2} \mathrm{O}\right)$, and partial Alveolar $\mathrm{CO}_{2}$ pressure $\left(\mathrm{PACO}_{2}\right)$ was used to estimate $\mathrm{PAO}_{2}$ from $\mathrm{FiO}_{2}$. $\mathrm{PiO}_{2}$ was defined by the ambient gas pressure $\left(\mathrm{P}_{a t m}\right)$ and the fraction of inspired $\mathrm{O}_{2}\left(\mathrm{FiO}_{2}\right)$ :

$$
\begin{aligned}
\mathrm{PAO}_{2}= & \mathrm{FiO}_{2} \cdot\left(\mathrm{P}_{\text {atm }}^{0}-\mathrm{PH}_{2} \mathrm{O}\right)-\frac{P A C O_{2}}{R Q}+ \\
& \mathrm{PACO}_{2} \cdot \mathrm{FiO}_{2} \cdot \frac{1-R Q}{R Q}
\end{aligned}
$$

where typically $\mathrm{PACO}_{2}=5.33 \mathrm{kPa}(40 \mathrm{mmHg})$ and $\mathrm{PH}_{2} \mathrm{O}=6.27 \mathrm{kPa}(47 \mathrm{mmHg})$ at $37^{\circ} \mathrm{C}$ and $\mathrm{RQ}=0.8$. To simplify calculations, we assumed that these parameters are fixed for the purpose of this study. We assumed standard ambient temperature $\mathrm{T}^{0}$ and pressure $\mathrm{P}_{a t m}^{0}$ at sea level. $\mathrm{FiO}_{2}$ was selected as a variable, ranging from $11 \%$ to $51 \%$. $\mathrm{FiO}_{2}$ above the ambient value of $21 \%$ corresponded to administration of oxygen. Decreasing $\mathrm{P}_{a t m}^{0}$ or reducing $\mathrm{FiO}_{2}$ values have a similar effect on $\mathrm{PAO}_{2}$, leading to a standardized oxygen concentration. Therefore $\mathrm{FiO}_{2}$ values below $21 \%$ were equivalent to the effect of decreasing $\mathrm{P}_{a t m}^{0}$, as would be the case for subjects living at higher altitude (equivalent altitude). The lowest used $\mathrm{FiO}_{2}=11 \%$ would correspond to an altitude of approximately $5000 \mathrm{~m}$ above sea level.

The $\mathrm{PxO}_{2}$ decreases between alveoli $\left(\mathrm{PAO}_{2}\right)$, end-capillaries $\left(\mathrm{Pc}^{\prime} \mathrm{O}_{2}\right)$ and systemic arteries $\left(\mathrm{PaO}_{2}\right)$. This difference in partial pressure $\mathrm{P}[\mathrm{A}-\mathrm{a}] \mathrm{O}_{2}$ is largely due to shunted blood and a mismatch of ventilation and perfusion $\dot{V}_{A} / \dot{Q}$, where $\dot{V}_{A}$ is the alveolar ventilation and $\dot{Q}$ is the blood flow. Under normal conditions, incomplete capillary diffusion is 0 and $\mathrm{PAO}_{2}=\mathrm{Pc}^{\prime} \mathrm{O}_{2}$ (West 2012). The inequality in oxygen content between end-capillary, venous and arterial blood is commonly referred to as VS. VS can be estimated from the shunted blood flow $\dot{Q}_{s}$ and total blood flow $\dot{Q}_{t}$ (West 2012):

$$
\begin{aligned}
V S & =\frac{\dot{Q}_{s}}{\dot{Q}_{t}}=\frac{\mathrm{Ccl} \mathrm{O}_{2}-\mathrm{CaO}}{\mathrm{Ccl} \mathrm{O}_{2}-\mathrm{C} \bar{v} \mathrm{O}_{2}} \\
& =\frac{\mathrm{CclO}_{2}-\mathrm{CaO}_{2}}{\mathrm{Ccl} \mathrm{O}_{2}-\mathrm{CaO}_{2}+\left(\mathrm{CaO}_{2}-\mathrm{C} \bar{v} \mathrm{O}_{2}\right)},
\end{aligned}
$$

where $\mathrm{CxO}_{2}$ is the oxygen content of blood, $\mathrm{C} c \mathrm{O}_{2}$ the end-capillary content, and typically $\left(\mathrm{CaO}_{2}\right.$ $\left.\mathrm{C} \bar{v} \mathrm{O}_{2}\right)=5 \mathrm{ml} / 100 \mathrm{ml}$ of blood. $\mathrm{CxO}_{2}$ can be obtained using

$$
\mathrm{CxO}_{2}=\frac{1.34 \cdot \mathrm{Hb} \cdot \mathrm{SxO}_{2}}{100}+0.003 \cdot \mathrm{PxO}_{2},
$$

where typically the oxygen-combining capacity of hemoglobin in blood is $1.34 \mathrm{ml} \mathrm{O} /(\mathrm{g} \mathrm{Hb}), \mathrm{Hb}=$ $12 \mathrm{~g} / 100 \mathrm{ml}$ is the hemoglobin concentration in blood, $\mathrm{SxO}_{2}$ is the oxygen saturation, 0.003 is the solubility of $\mathrm{O}_{2}$ in blood in $\frac{\mathrm{ml} \mathrm{O}_{2}}{100 \mathrm{ml} \cdot 1 \mathrm{mmHg}}$.

$\mathrm{VS}$ is important to the clinician as its magnitude describes the efficiency of gas exchange in the patient which would represent the severity of the underlying pathological disease process. Therefore, 
we use VS as an output variable that is estimated by the mathematical model. Since Eq. 4 and 5 can only be solved numerically, this may be inefficient to apply to a pulse oximeter. An alternative is to use $\mathrm{P}[\mathrm{A}-\mathrm{a}] \mathrm{O}_{2}$ as an estimate of VS with the inherent limitations of using partial pressure rather than oxygen content. This measure describes the gradient of $\mathrm{PO}_{2}$ due to the underlying pathology and can be easily displayed in a oxygen cascade (Figure 2). When calculating $\mathrm{VS}, \mathrm{PaO}_{2}$ is not estimated from $\mathrm{FiO}_{2}$ but from $\mathrm{SaO}_{2}$.

Hill's equation has been modified by Severinghaus to calculate $\mathrm{SxO}_{2}$ from $\mathrm{PxO}_{2}$ (Severinghaus 1979):

$$
\mathrm{SxO}_{2}=\frac{1}{\frac{23400}{\mathrm{PxO}_{2}{ }^{3}+150 \cdot \mathrm{PxO}_{2}}+1} .
$$

The Severinghaus - Ellis equations (Severinghaus 1979, Ellis 1989) allow for the conversion of $\mathrm{SxO}_{2}$ to $\mathrm{PxO}_{2}$ :

$$
\mathrm{PxO}_{2}=(B+A)^{1 / 3}-(B-A)^{1 / 3}
$$

where

$$
\begin{aligned}
A & =\frac{11700}{\frac{1}{S x O_{2}}-1}, \\
B & =\sqrt{50^{3}+A^{2}} .
\end{aligned}
$$

Equations 6 and 7 describe the nonlinear S-shaped $\mathrm{Hb}-\mathrm{O}_{2}$ dissociation curve in both directions (Figure 3). As the term $\mathrm{A}$ of equation 8 is not defined for $\mathrm{SxO}_{2}=100 \%$, we replaced $100 \%$ with $99.9 \%$ in our model calculations.

In clinical practice $\mathrm{SaO}_{2}$ is either obtained by analyzing an arterial blood sample in a blood gas analyzer or when not available, estimated using $\mathrm{SpO}_{2}$ from the pulse oximeter reading. The $\mathrm{A}_{R M S}$ error in this $\mathrm{SaO}_{2}$ estimation (as illustrated in Figure 1) is propagated in our model using the SeveringhausEllis equation 7 . The pulse oximeter can be considered as an additional stage in the oxygen cascade and $\mathrm{SpO}_{2}$ is used as an input variable that can be measured in the clinical setting (Figure 2).

\subsection{Experiments}

After obtaining ethics approval and written consent, 19 healthy non-smoking subjects (11 males, 8 females, median age 28, range 19 to 50 years) with no history of cardio-respiratory or neurological disease were recruited for a controlled desaturation study. The primary aim of the study was to record data for calibration experiments of a novel mobile phone pulse oximeter using a camera (Karlen et al. 2012). The subjects were invited to the physiology laboratory where they rested for 5 min and then underwent a health check by measuring baseline blood pressures, heart rate and $\mathrm{SpO}_{2}$. Fitzpatrick Skin Phototype (Fitzpatrick et al. 1988) was assessed (median III, range II to V). Following the successful health check, the study sensors were applied and recording initiated. After 20 min of recording at sea level $\left(\mathrm{FiO}_{2}=21 \%\right)$, each subject entered a normobaric hypoxia chamber with a $\mathrm{FiO}_{2}$ set to 


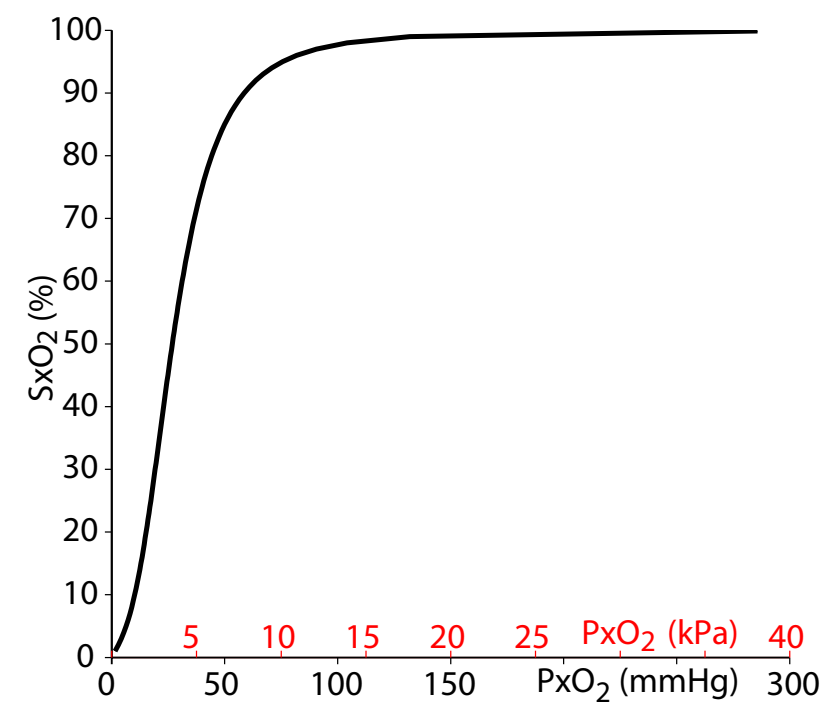

Figure 3: The $\mathrm{Hb}-\mathrm{O}_{2}$ dissociation curve describes the non-linear relationship between $\mathrm{PxO}_{2}$ and $\mathrm{SxO}_{2}$ and is obtained using the Severinghaus-Ellis equations. This characteristic is largely due to a change in $\mathrm{O}_{2}$ affinity for hemoglobin. 


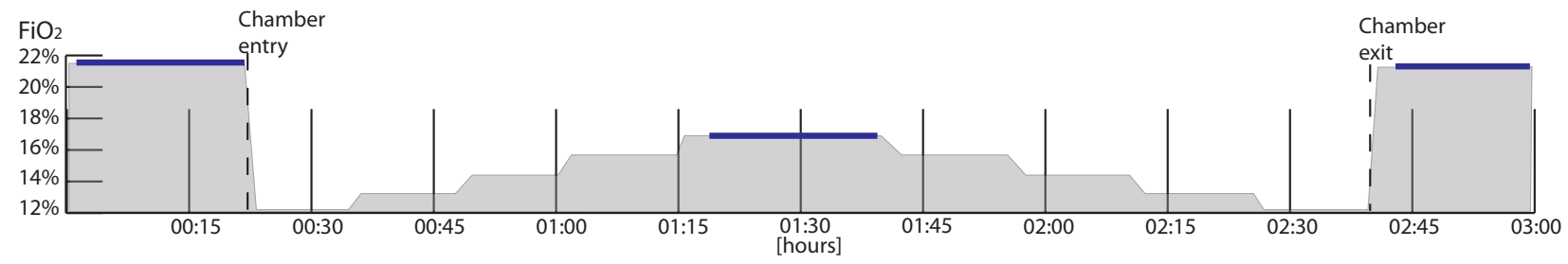

Figure 4: Sequence of $\mathrm{FiO}_{2}$ changes in the hypoxia chamber during the experiment. Duration is approximate as stabilization of $\mathrm{FiO}_{2}$ is dependent on subject in chamber. The blue bold lines depict the data collected for the analysis in this manuscript.

$12 \%$. The $\mathrm{FiO}_{2}$ was then increased stepwise to $17 \%$ and then again reduced to $12 \%$ (Figure 4 ). After approximately 2.5 hours in the chamber, when an $\mathrm{FiO}_{2}=12 \%$ was attained and maintained for 5 min, the subjects exited the chamber and were monitored for another $20 \mathrm{~min}$ at $\mathrm{FiO}_{2}=21 \%$.

The study sensors consisted of four pulse oximeters that recorded reference data simultaneously. Both, the dominant and non-dominant hands of each subject were fitted with a Nonin Xpod 16bit (NON, Nonin Medical Inc, Plymouth, USA) and a Masimo Low Power Module (MAS, Masimo Corp, Irvine, USA) oximeter module and finger probe, each connected to a Phone Oximeter on an iPod Touch (Apple Inc, USA) (Karlen et al. 2011). The devices recorded a photoplethysmogram (16 bit; MAS 62.5 $\mathrm{Hz}$, NON $75 \mathrm{~Hz})$, heart rate $(1 \mathrm{~Hz})$, and $\mathrm{SpO}_{2}(1 \mathrm{~Hz})$. They were configured to calculate $\mathrm{SpO}_{2}$ over an average of 8 beats for NON and $8 \mathrm{~s}$ for MAS respectively. Both brands of pulse oximeters also provided indicators of artifacts, low perfusion and sensor problems. The sensor data sheets reported an $\mathrm{A}_{R M S}$ of \pm 2 digits for NON and $\pm 2 \%$ for MAS under conditions without artifacts. Inspired and expired $\mathrm{O}_{2}$ and $\mathrm{CO}_{2}$ and respiratory rate were recorded using Datex-Ohmeda S5 Collect (GE Healthcare, Fairfield, USA) hardware and software at $1 \mathrm{~Hz}$. All recording devices were synchronized to a common time server and a marker was pressed at the beginning of the experiment to verify synchronization.

All data from the reference oximeters on the non-dominant hand and the gas monitor were paired for this study. The data from the dominant hand were not used as they were subject to an increased level of artifacts that would potentially contaminate the data for the purposes of this study. Data pairs labeled with an artifact, low perfusion (MAS perfusion index $<1$ or $>18$, NON "red" perfusion) or sensor warnings of either oximeter were eliminated. The first three minutes after entering or exiting the chamber and after changing $\mathrm{FiO}_{2}$ inside the chamber, where subjects were exposed to large changes in $\mathrm{FiO}_{2}$ in a short period of time, were also discarded to eliminate variations in response time effects of the oximeters and individual subjects physiology.

The distribution of the $\mathrm{SpO}_{2}$ values for a given pulse oximeter at an $\mathrm{FiO}_{2}$ of $21 \% \pm 0.3$ and $17 \% \pm 0.3$ were plotted as histograms for each device. The $17 \%$ threshold was chosen as a second level because it provided the second largest count of data pairs and contained the data with least adaptation effects (changes in respiratory rate and end tidal $\mathrm{CO} 2$ ). Also, the expected $\mathrm{SpO}_{2}$ for this $\mathrm{FiO}_{2}$ is in the range of $93 \%-94 \%$, which coincides with the commonly used threshold for clinical decision 
making. It was therefore particularly interesting to analyze pulse oximeter tolerance for that range. The standard deviation (SD) for all subjects and the mean of the intra-subject standard deviation (iSD) were calculated. iSD is a more conservative measure as it disregards inter-subject variations that might be present due to sensor placement, perfusion, physiology or other reasons that would lead to measurement variations between observations in different subjects. The obtained distributions were compared with the probability density functions obtained by the model for an 'ideal' pulse oximeter with $\mathrm{A}_{R M S}=2 \%$. The probability density functions were plotted for subjects with VSs of $0 \%, 2 \%$ and $5 \%$, all considered normal levels.

\section{Results}

Propagation of the $\mathrm{A}_{R M S}$ through the Severinghaus-Ellis equations $(7,8)$ reveals that due to the nonlinear relationship of $\mathrm{SaO}_{2}$ and $\mathrm{PaO}_{2}$ the effect of pulse oximeter tolerance is more important at higher $\mathrm{SpO}_{2}$ (Figure 5, top). Above $\mathrm{SpO}_{2}=95 \%$, the spread of $\mathrm{PaO}_{2}$ is so large that based on a single observed $\mathrm{SpO}_{2}$, estimation of $\mathrm{PaO}_{2}$ is highly unreliable. In contrast, the tolerance does not cause a large variation in the estimation of virtual shunt (Figure 5, bottom).

\subsection{Oxygen Cascade Model}

The oxygen cascade predicts the maximal $\mathrm{PaO}_{2}$ for a healthy subject at $\mathrm{FiO}_{2}=21 \%$ (Figure 6). The maximal $\mathrm{PaO}_{2}$ with a VS of $0 \%\left(\mathrm{PAO}_{2}=\mathrm{PaO}_{2}\right)$ and $\mathrm{FiO}_{2}=21 \%$ was $98 \mathrm{mmHg}(13 \mathrm{kPa})$ at sea level and consequently, when the Severinghaus-Ellis equations were applied, the $\mathrm{SaO}_{2}$ was $97.8 \%$. The oxygen cascade demonstrates that $\mathrm{PAO}_{2}$ increases linearly with an increase in $\mathrm{FiO}_{2} \cdot \mathrm{PaO}_{2}$ estimated from $\mathrm{SpO}_{2}$ showed a non-linear relationship characterized by the Severinghaus-Ellis equations. and $\mathrm{PaO}_{2}$ estimates. Due to the precision of pulse oximeters, depicted in green (68\% percentile) error bars, the estimated $\mathrm{PaO}_{2}$ can be larger than the maximal possible value $\left(\mathrm{PAO}_{2}\right)$ and consequently VS is negative. The graph in Figure 5 allows for an estimate of the likelihood of a shunt for a given $\mathrm{SpO}_{2}$ observation. For example, a measured $\mathrm{SpO}_{2}$ of $94 \%$ (black filled dot) has an $84 \%$ confidence to have a virtual shunt between $7.5 \%$ and $19 \%$.

\subsection{Experiments}

A total of 32,672 sample points were obtained for $\mathrm{FiO}_{2}=21 \%$ and 6,085 for $\mathrm{FiO}_{2}=17 \%$ (Table 1). The $\mathrm{SpO}_{2}$ distributions were different for the two pulse oximeter brands at $\mathrm{FiO}_{2}=21 \%$ (Figure 7 ) and $\mathrm{FiO} 2=17 \%$ (Figure 8). Both sensors were within range when comparing the reported SD and iSD values to the specified accuracy in the respective data sheet. SD was larger than iSD in all situations (Table 1), indicating variability between individual subjects. This variability can be also observed in Figure 8 where a second peak in the NON distribution can clearly be identified. This variability was also present for the $\mathrm{FiO}_{2}=21 \%$, but was not sufficiently large to produce a secondary peak. There was 

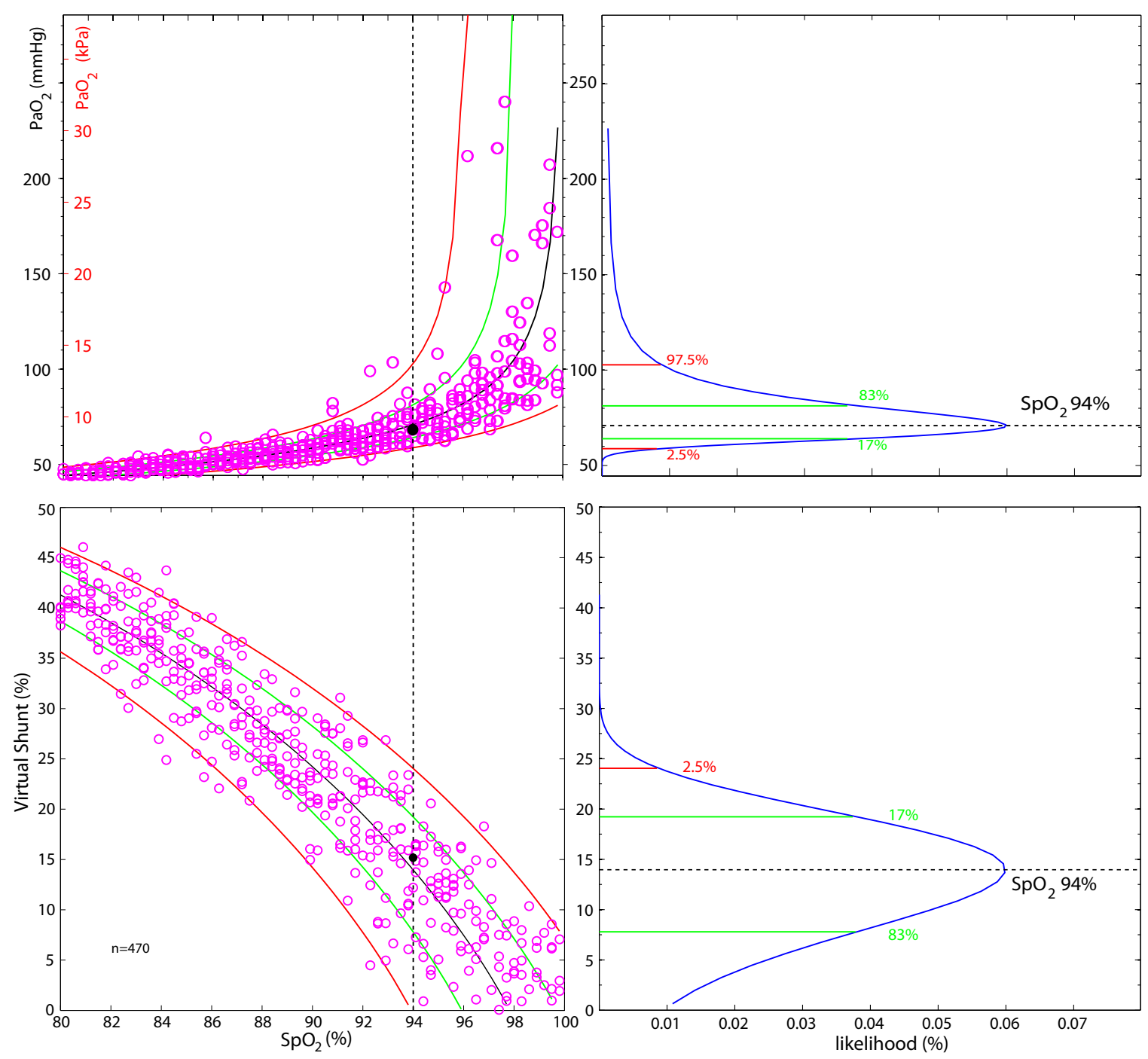

Figure 5: The simulated relationship between oxygen saturation measured by pulse oximetry $\left(\mathrm{SpO}_{2}\right)$ compared to systemic arterial oxygen partial pressure $\left(\mathrm{PaO}_{2}\right.$, top) and virtual shunt (VS, bottom). The scatterplots were obtained by applying the Severinghaus-Ellis equation to the simulated data from Figure 1. An approximately logarithmic relationship between measured $\mathrm{SpO}_{2}$ and estimated $\mathrm{PaO}_{2}$ was obtained. Pulse oximeter tolerances had a greater effect on $\mathrm{PaO}_{2}$ at a high $\mathrm{SpO}_{2}$ due to the flat shape of the $\mathrm{Hb}-\mathrm{O}_{2}$ dissociation curve in this range (94\% to 100\%). This effect is reduced in the calculation of VS, since the tolerances of the pulse oximeter only apply to the dissolved oxygen portion of the shunt equation. The pulse oximeter tolerances (confidence intervals of accuracy) are represented by the green $(68 \%)$ and red (95\%) lines. 

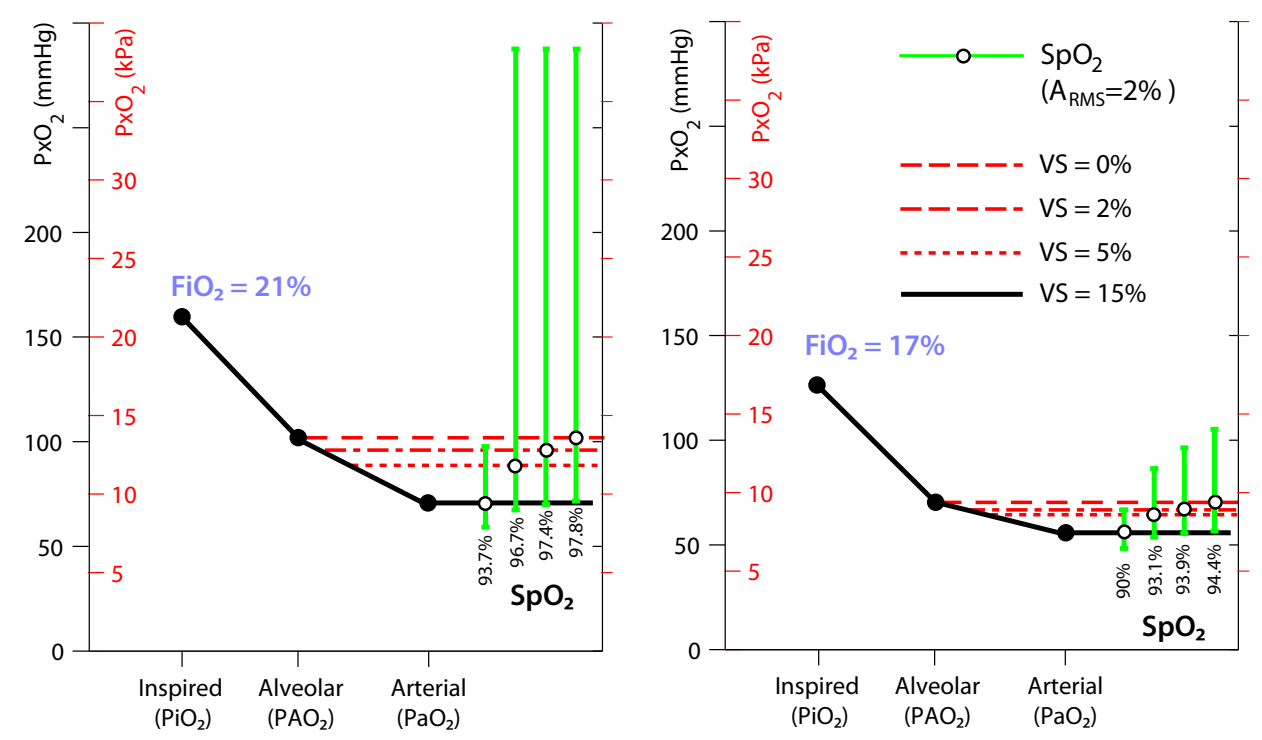

Figure 6: Oxygen cascades for $\mathrm{FiO}_{2}=21 \%$ (left) and $17 \%$ (right) obtained from our theoretical model showing $\mathrm{PxO}_{2}$ for the inspired, alveolar and arterial stages at a virtual shunt (VS) of $15 \%$ (black). The horizontal dashed lines represent alternative $\mathrm{PaO}_{2}$ for VS $=0 \%, 2 \%$ and $5 \%$ respectively. The pulse oximeter (with $\mathrm{A}_{R M S}=2 \%$ ) stage is overlaid over the arterial stage for each VS level. The tolerance intervals of the pulse oximeter, showing the measurement $68 \%$ confidence interval (green error bar). High $\mathrm{SpO}_{2}$ values have large tolerance intervals due to flat portion of the $\mathrm{Hb}-\mathrm{O}_{2}$ dissociation curve. Independent of $\mathrm{FiO}_{2}$, the confidence interval of the $\mathrm{SpO}_{2}$ reading for $\mathrm{VS}=0 \%$ overlaps with a potential reading for $\mathrm{VS}=15 \%$ (the most right $\mathrm{SpO}_{2}$ ). 


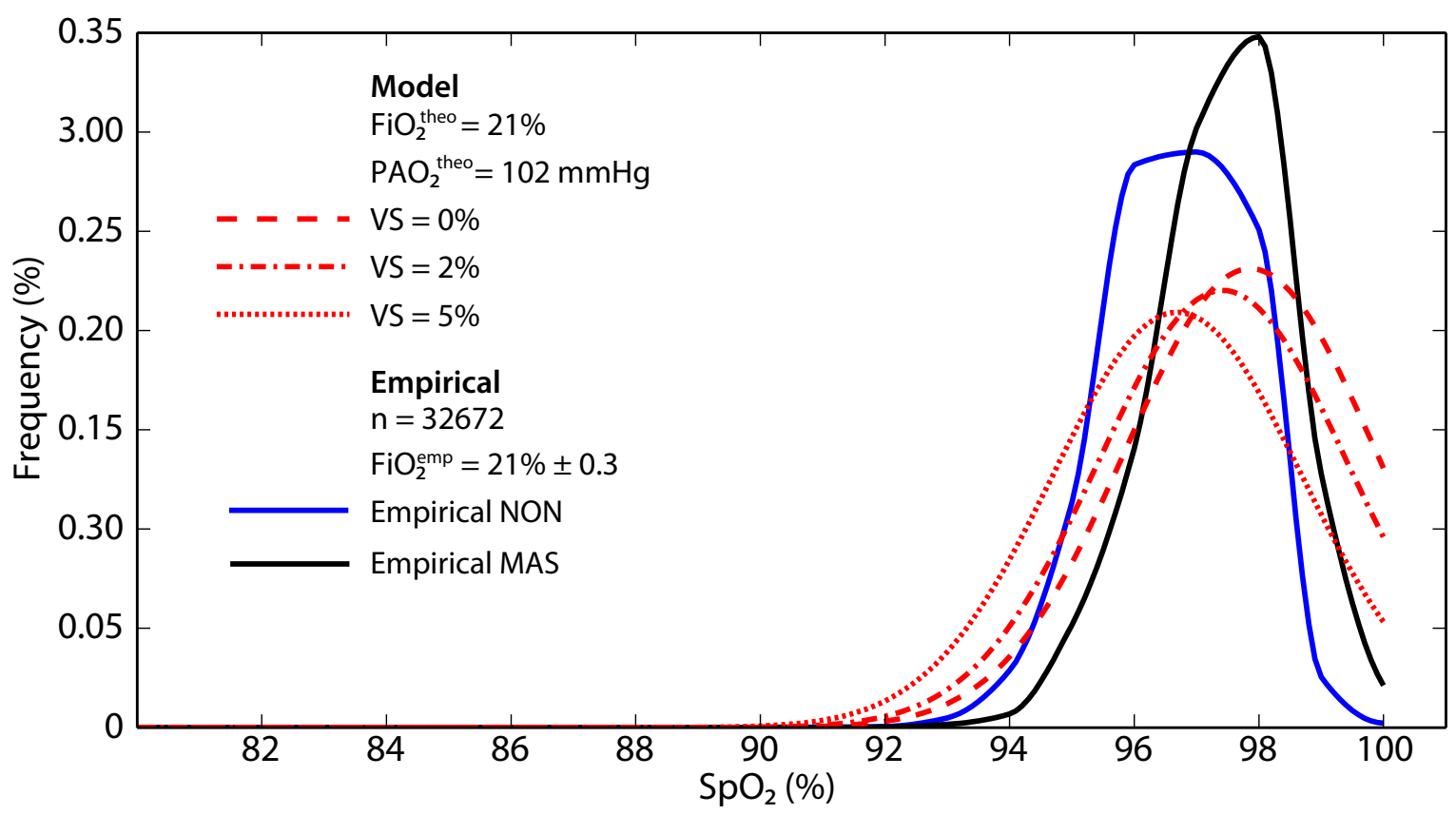

Figure 7: Comparison of probability density functions of the model with the empirical distributions of pulse oximeter readings for $\mathrm{FiO}_{2}=21 \%$. Empirical data was obtained for two pulse oximeter brands, NON (blue) and MAS (black), recording from 19 subjects at rest at sea level. Probability density functions for a pulse oximeter with $\mathrm{A}_{R M S}=2 \%$ were calculated for virtual shunts $(\mathrm{VS})=0,2$, and $5 \%$ ( red dashed and dotted lines). The pulse oximeters distributions from both devices fit within the theoretical distributions, despite the fact that there is a visible bias between the two empirical distributions.

a clear bias of $1.2 \%$ between the oximeters at an $\mathrm{FiO}_{2}=21 \%$, which increased to $2.1 \%$ at an $\mathrm{FiO} 2=$ $17 \%$ (Table 1).

\section{Discussion}

We have described a model to estimate $\mathrm{VS}$ from $\mathrm{FiO}_{2}$ and $\mathrm{SpO}_{2}$. The model facilitates the analysis of effects of variability in pulse oximeter readings due to tolerances in the $\mathrm{SaO}_{2}$ estimations. An improved clinical understanding of the limitations of pulse oximetry will promote improved clinical decision making.

The VS has been used as estimate of the pathological severity of an impairment of oxygen delivery. This theoretical concept describes the overall loss of $\mathrm{O}_{2}$ content between the alveolar gas and arterial blood. Estimating VS is of clinical importance as its magnitude can be used to estimate disease 


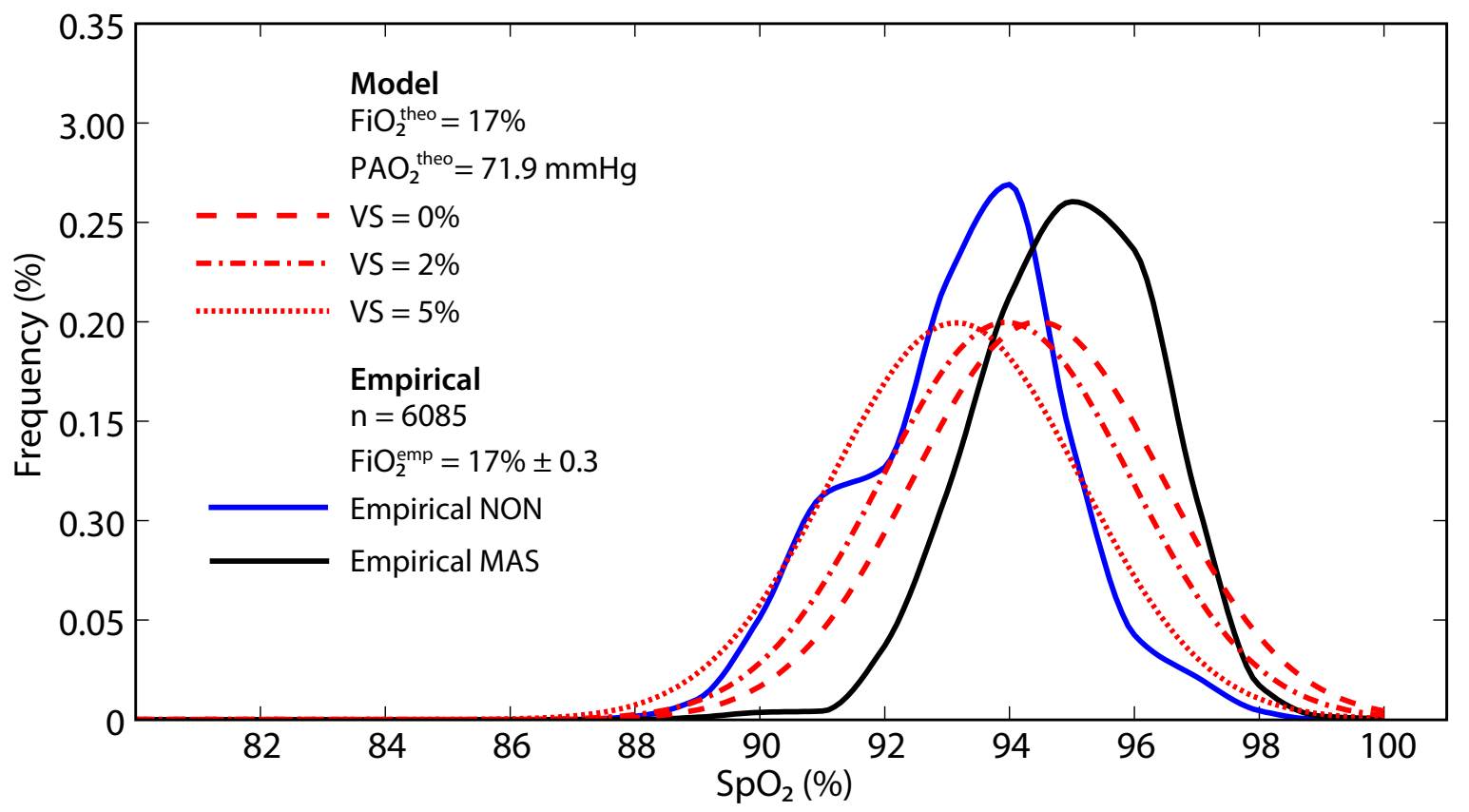

Figure 8: Comparison of probability density functions of the model with the empirical distributions of pulse oximeter readings for $\mathrm{FiO}_{2}=17 \%$. Empirical data was obtained from two pulse oximeter brands, NON (blue) and MAS (black), from 19 subjects at rest in a normobaric hypoxia chamber. Probability density function for a pulse oximeter with $\mathrm{A}_{R M S}=2 \%$ were calculated for virtual shunts (VS) $=0$, 2, and 5\% ( red dashed and dotted lines). Pulse oximeters distributions from both devices fit within the theoretical distributions, despite the fact that there is a visible bias between the two empirical distributions.

Table 1: The distributions of oximeter measurements obtained from the hypoxic chamber experiments. Blood oxygen saturation $\left(\mathrm{SpO}_{2}\right)$ and heart rate (HR) was collected for two inspired gas fractions $\left(\mathrm{FiO}_{2}\right)$ and two pulse oximeter brands (NON and MAS). Mean, standard deviation (SD) and bias between oximeters were calculated for $\mathrm{n}$ data pairs combined from the 19 subjects. The mean from the intra-subject SD (iSD) is also reported. iSD is more accurate than SD in describing the sensor tolerance.

\begin{tabular}{|c|c|c|c|c|c|c|c|c|c|c|c|c|c|}
\hline \multirow[t]{3}{*}{$\mathrm{FiO}_{2}(\%)$} & \multicolumn{7}{|c|}{$\mathrm{SpO}_{2}(\%)$} & \multicolumn{5}{|c|}{ HR (beats/min) } & \multirow[t]{3}{*}{$\mathrm{n}^{1}$} \\
\hline & \multicolumn{3}{|c|}{$\mathrm{NON}$} & \multicolumn{3}{|c|}{ MAS } & \multirow[b]{2}{*}{ Bias } & \multicolumn{2}{|c|}{$\mathrm{NON}$} & \multicolumn{2}{|c|}{ MAS } & \multirow[b]{2}{*}{ Bias } & \\
\hline & Mean & $\mathrm{SD}$ & iSD & Mean & $\mathrm{SD}$ & iSD & & Mean & $\mathrm{SD}$ & Mean & $\mathrm{SD}$ & & \\
\hline $21 \pm 0.3$ & 96.7 & 1.2 & 0.8 & 97.8 & 1.1 & 0.7 & 1.2 & 73.3 & 9.8 & 73.1 & 9.9 & -0.3 & 32672 \\
\hline $17 \pm 0.3$ & 93.2 & 1.7 & 1.1 & 95.4 & 1.4 & 1.0 & 2.1 & 72.4 & 9.5 & 72.1 & 9.5 & -0.3 & 6085 \\
\hline
\end{tabular}

\footnotetext{
${ }^{1}$ The samples are not statistically independent as averaging is used during sampling
} 
severity and, more importantly, to track the progression of the process over time. It can also be used to optimize therapies such as ventilation and oxygen administration. However, measuring VS directly is not clinically practical. Typically, graphs containing iso-shunt lines are used by clinicians to estimate VS based on known $\mathrm{FiO}_{2}-\mathrm{PaO}_{2}$ relationships (Benatar et al. 1973). Alternatively, VS has been modeled in a computer simulation and can be estimated by entering $\mathrm{FiO}_{2}-\mathrm{PaO}_{2}$ pairs into the software interface (Kretschmer et al. 2013). Exact $\mathrm{PaO}_{2}$ is available through blood gas analysis, which can only be accessed with delays at discreet time intervals. A potential application of pulse oximetry would be to indirectly estimate the magnitude of VS continuously by estimating $\mathrm{SaO}_{2}$ and $\mathrm{PaO}_{2}$. Our model expands the concept of $\mathrm{PiO}_{2}$ vs. $\mathrm{SpO}_{2}$ diagrams (Sapsford \& Jones 1995) by displaying pulse oximeter tolerances. The concept of tolerance intervals has been used since the beginning of pulse oximetry to describe the accuracy and limitations of pulse oximeters (Wukitsch et al. 1988). However, it has rarely been used for direct display in oximeters or estimations of shunt.

The simulated experiments show that the non-linearity of the $\mathrm{Hb}-\mathrm{O}_{2}$ dissociation curve (Figure 3) cause large effects in the $\mathrm{PaO}_{2}$ estimation at high $\mathrm{SpO}_{2}$ when taking pulse oximeter tolerance into account. This high variability in estimated $\mathrm{PaO}_{2}$ over a short range of $\mathrm{SpO}_{2}$ can be misleading due to the flat portion of the $\mathrm{Hb}-\mathrm{O}_{2}$ dissociation curve. However, since the reported error rate of pulse oximeters do not vary over a larger range (typically 80\%-100\%), the estimation of $\mathrm{PaO}_{2}$ from pulse oximeter readings becomes more accurate at a lower $\mathrm{SpO}_{2}$. In an analogous situation, a small change in the oxygen saturation on the flat portion of the oxygen dissociation curve would indicate a much bigger change in $\mathrm{PaO}_{2}$ than the same amount of change at a lower saturation. The true $2 \%$ change in $\mathrm{SaO}_{2}$ in the high nineties indicates a much more significant change in pathology than the $2 \%$ change in the patient with a $\mathrm{SaO}_{2}$ in the low eighties. The effects on the estimation of virtual shunt is more linear than $\mathrm{PaO}_{2}$ (Figure 5). This is explained by the fact that the tolerances of the pulse oximeter only apply to the dissolved oxygen portion of the equation. However, the model shows that large oximeter tolerances can cause variations of up to $25 \%$ at a $\mathrm{SpO}_{2}=94 \%$.

From the theoretical models it becomes clear that at sea level, an $\mathrm{SaO}_{2}$ of $98 \%$ and higher is only possible if $\mathrm{O}_{2}$ is administered. The experimental data confirmed this, with peaks in the $\mathrm{SpO}_{2}$ distribution at $98 \%(\mathrm{MAS})$ and $97 \%(\mathrm{NON})$. At this altitude, an ideal pulse oximeter with $\mathrm{A}_{R M S}=$ $2 \%$ could display any $\mathrm{SpO}_{2}$ between $96 \%$ and $100 \%$ at a $68 \%$ tolerance interval or $94 \%$ to $100 \%$ at a 95\% tolerance interval. This corresponds to a maximal estimated VS of $7.5 \%$ and $14 \%$ respectively. To produce an $\mathrm{SaO}_{2}$ of $100 \%$ in a patient at sea level, the administration of at least $47 \% \mathrm{O}_{2}$ would theoretically be required.

An increase in bias for $\mathrm{SpO}_{2}$ values lower than $95 \%$ between oximeters has been previously observed (Van de Louw et al. 2001). Both tested sensors were within tolerance specified in their data sheets when comparing the reported values to the theoretical values of an ideal oximeter that complies with ISO standards. NON reported more conservative $\mathrm{SpO}_{2}$ values than the MAS, suggesting a larger VS $(\approx 5 \%)$. The distribution of MAS values was very close to the theoretical maximum achievable $\mathrm{SpO}_{2}$, corresponding to a VS $=0 \%$ suggesting that this type of sensor may be overestimating $\mathrm{SaO}_{2}$.

Our theoretical and practical findings show that the accurate estimation of $\mathrm{PaO}_{2}$ and VS is challenging with a single $\mathrm{SpO}_{2}$ measurement as the tolerance of pulse oximeters is relatively large $\left(\mathrm{A}_{R M S}\right.$ 
$=2 \%$ ). An oxygen cascade graph can easily illustrate this inaccuracy and the effect on VS estimation (Figure 6). Oximeter readings suggesting a VS of $0 \%$ cannot exclude a VS of $15 \%$ at the $68 \%$ confidence interval. This uncertainty may be reduced with collecting multiple independent samples (ideally under different conditions) that will describe an $\mathrm{SaO}_{2}$ distribution. Changing conditions can be produced in various ways. To establish their model, Kjaergaard et al. (2003) administered $\mathrm{O}_{2}$ to patients to obtain a variation in exhaled $\mathrm{O}_{2}$ and subsequently calibrate the model. In addition they relied on a invasive blood sample to calibrate for $\mathrm{Hb}$ and $\mathrm{pH}$. As these invasive measures were not available in our setting, we relied on multiple observations of the same subject state with two independent oximeters. These observations result in a distribution with the mean describing the most likely estimation of $\mathrm{SaO}_{2}$. Such an approach is already practiced with automated blood pressure devices that repeat measurements 3 to 5 times and report an average to minimize measurement error and white-coat effects (Myers 2006). However, the use of multiple measurement points of a continuous time series, without replacing or relocating the oximeter probe (as performed with the blood pressure device) would not reduce the overall error. Pulse oximeters use an internal, proprietary averaging algorithm making measurements dependent on previous samples. Collecting multiple $\mathrm{SpO}_{2}$ samples is already manually performed in practice when the measurement procedure is repeated with repositioning or placement of a different sensor. This is produced when the results of the initial measurement do not seem plausible, e.g. when the user suspects an incorrectly placed or defective probe. However, there is a need for performing this measurement repetition in a more systematic way which needs further investigation. In addition, the method for merging the observations (i.e. mean or median) and the number of multiple, consecutive $\mathrm{SpO}_{2}$ measurements required to provide the best estimate of $\mathrm{SaO}_{2}$ with higher accuracy needs investigation. Finally, technical improvements in the oximeter device itself could provide direct guidance for the need to repeat the measurements at specific intervals and sensor locations.

\subsection{Limitations}

In this work, a number of co-variates in the model such as $\mathrm{Hb}$ and $\mathrm{PxCO}_{2}$ were kept invariant. While our physiological models would allow these parameters to be included as variables in the theoretical calculations, exact values are not available in clinical practice without invasive sampling and were also not available during our experiments. Our model consists of a single compartment to estimate VS. More precise models, for example with two (Kretschmer et al. 2013, Karbing et al. 2011), three (Jones \& Jones 2000, Karbing et al. 2011) and five (Wang et al. 2010) compartments, have been suggested. However, considering the shown tolerance effects of pulse oximeters on the models overall accuracy, the increased complexity of higher compartment order is unlikely improving VS estimations in practice.

A low $\mathrm{PaO}_{2}$ triggers a physiological response that compensates for the reduced $\mathrm{O}_{2}$ intake during breathing of hypoxic gas mixes. A short term adaptation is the increase of respiratory drive and consequent reduction of $\mathrm{PACO}_{2}$. This is a typical adaptation to higher altitudes and is also observed in our data. $\mathrm{PACO}_{2}$ and all other $\mathrm{CO}_{2}$ partial pressures will decrease immediately at exposure causing a left shift of the $\mathrm{Hb}-\mathrm{O}_{2}$ dissociation curve and a relative increase in $\mathrm{PAO}_{2}$. This could not be avoided during data collection, but was minimized by using data for analysis that was collected after the 
typical short term adaptation period of 3 minutes. The longer term (multiple hours/days) adaptation process of increasing the concentration of 2,3-diphosphoglycerate leading to a right shift of the $\mathrm{Hb}-\mathrm{O}_{2}$ dissociation curve and lowering the $\mathrm{O}_{2}$ affinity (West 2012) was considered negligible due to the short duration of our experiments.

For this study, arterial blood gas samples were not available to confirm the estimated $\mathrm{SaO}_{2}$. We can therefore only speculate about the real $\mathrm{PaO}_{2}$ and VS that the subjects experienced during the experiments. This corresponds to many clinical situations where arterial sampling of blood gases is not immediately available or desirable.

\section{Conclusion}

We have illustrated the propagation of pulse oximeter tolerance from the measurement of $\mathrm{SpO}_{2}$ to the estimation of VS. Theoretical and experimental results show that the effect is amplified at higher $\mathrm{SpO}_{2}$ readings. The findings show that the estimation of $\mathrm{PaO}_{2}$ and $\mathrm{VS}$ should ideally not be made from a single $\mathrm{SpO}_{2}$ observation. To reduce tolerance effects and artifacts due to aberrant oximeter readings in the clinical setting we recommend performing multiple observations of $\mathrm{SpO}_{2}$ before undertaking critical clinical decisions.

\section{Acknowledgements}

The authors are grateful to Prof. Michael Koehle, Prof. Jim Rupert and Martin MacInnis at the School of Kiniesology, The University of British Columbia, Vancouver for making the hypoxia chamber available for our research and all volunteers that participated in the experiments. We are also thankful to Dr. Heng Gan, Dorothy Meyers, and Aryannah Umedaly who kindly helped us in conducting the data collection for this study. This work was financially supported in part by Grand Challenges Canada (CRS2_0041) and the Swiss National Science Foundation (PA00P2_136454).

\section{References}

Benatar, S. R., Hewlett, A. M. \& Nunn, J. F. (1973), 'The use of iso-shunt lines for control of oxygen therapy', Brit. J. Anaesth. 45, 711-8.

Ellis, R. K. (1989), 'Determination of PO2 from saturation.', Journal of applied physiology (Bethesda, Md. : 1985) 67(2), 902.

Fitzpatrick, T., Types, S.-r. S. \& Vi, I. T. (1988), 'The validity and practicality of sun-reactive skin types I through VI', Archives of Dermatology 124(6), 869-71. 
Gehring, H., Duembgen, L., Peterlein, M., Hagelberg, S. \& Dibbelt, L. (2007), 'Hemoximetry as the "gold standard"? Error assessment based on differences among identical blood gas analyzer devices of five manufacturers.', Anesthesia and analgesia 105(6 Suppl), S24-30.

ISO (2011), 'ISO 80601-2-61 Medical electrical equipment Part 2-61: Particular requirements for basic safety and essential performance of pulse oximeter equipment'.

Jones, J. G. \& Jones, S. E. (2000), 'Discriminating between the effect of shunt and reduced VA/Q on arterial oxygen saturation is particularly useful in clinical practice.', Journal of clinical monitoring and computing 16(5-6), 337-50.

Jubran, A. (1999), 'Pulse oximetry', Critical care 3(2), R11-R17.

Karbing, D. S., Kjæ rgaard, S. r., Andreassen, S., Espersen, K. \& Rees, S. E. (2011), 'Minimal model quantification of pulmonary gas exchange in intensive care patients.', Medical engineering $\& 3$ physics 33(2), 240-8.

Karlen, W., Dumont, G. A., Petersen, C., Gow, J., Lim, J., Sleiman, J. \& Ansermino, J. M. (2011), Human-centered Phone Oximeter Interface Design for the Operating Room, in V. Traver, A. Fred, J. Filipe \& H. Gamboa, eds, 'Proceedings of the International Conference on Health Informatics', SciTePress - Science and and Technology Publications, Rome, Italy, pp. 433-8.

Karlen, W., Lim, J., Ansermino, J. M., Dumont, G. \& Scheffer, C. (2012), Design challenges for camera oximetry on a mobile phone, in 'Annual International Conference of the IEEE Engineering in Medicine and Biology Society.', pp. 2448-51.

Kjaergaard, S. r., Rees, S., Malczynski, J., Nielsen, J. r. A., Thorgaard, P., Toft, E. \& Andreassen, S. (2003), 'Non-invasive estimation of shunt and ventilation-perfusion mismatch.', Intensive care medicine 29(5), 727-34.

Kretschmer, J., Becher, T., Riedlinger, A., Schädler, D., Weiler, N. \& Möller, K. (2013), A simple gas exchange model predicting arterial oxygen content for various FiO2 levels, in 'Annual International Conference of the IEEE Engineering in Medicine and Biology Society', pp. 465-8.

Mannheimer, P. D. (2007), 'The light-tissue interaction of pulse oximetry.', Anesthesia and analgesia 105(6 Suppl), S10-7.

Myers, M. G. (2006), 'Automated blood pressure measurement in routine clinical practice.', Blood pressure monitoring 11(2), 59-62.

Petros, A. J., Doré, C. J. \& Nunn, J. F. (1994), 'Modification of the iso-shunt lines for low inspired oxygen concentrations', British Journal of Anaesthesia 72(5), 515-22.

Sapsford, D. J. \& Jones, J. G. (1995), 'The PIO2 vs. SpO2 diagram: a non-invasive measure of pulmonary oxygen exchange.', European journal of anaesthesiology 12(4), 375-86. 
Severinghaus, J. W. (1979), 'Simple, accurate equations for human blood O2 dissociation computations.', Journal of applied physiology: respiratory, environmental and exercise physiology 46(3), 599602 (Revised version 2007).

Van de Louw, A., Cracco, C., Cerf, C., Harf, A., Duvaldestin, P., Lemaire, F. \& Brochard, L. (2001), 'Accuracy of pulse oximetry in the intensive care unit.', Intensive care medicine 27(10), 1606-13.

Wang, A., Mahfouf, M., Mills, G. H., Panoutsos, G., Linkens, D. A., Goode, K., Kwok, H. F. \& Denaï, M. (2010), 'Intelligent model-based advisory system for the management of ventilated intensive care patients: Hybrid blood gas patient model.', Computer methods and programs in biomedicine 99(2), 195-207.

Webb, R. K., Ralston, A. C. \& Runciman, W. B. (1991), 'Potential errors in pulse oximetry. II.', Anaesthesia 46(3), 207-12.

Weininger, S. (2007), 'Effective standards and regulatory tools for respiratory gas monitors and pulse oximeters: the role of the engineer and clinician.', Anesthesia and analgesia 105(6 Suppl), S95-9.

West, J. B. (2012), Respiratory Physiology, 9 edn, Lippincott Williams \& Wilkins, Baltimore.

Wukitsch, M. W., Petterson, M. T., Tobler, D. R. \& Pologe, J. A. (1988), 'Pulse oximetry: Analysis of theory, technology, and practice', Journal of Clinical Monitoring 4(4), 290-301. 